\section{Implication d'Angpt|4 dans le syndrome néphrotique}

\section{Une protéine à deux visages}

Camille Macé, Lionel C. Clément
Glomerular Disease Therapeutics Laboratory, University of Alabama at Birmingham, UAB Division of Nephrology, THT 616, 1900 University Boulevard, Birmingham AL 35294, États-Unis.

Iclement@uab.edu
Définition du syndrome néphrotique Le syndrome néphrotique est une maladie rénale touchant à l'intégrité structurelle et/ou fonctionnelle du glomérule. Le filtre glomérulaire est constitué de cellules épithéliales (les podocytes), d'un système endothélial capillaire fenêtré et d'une membrane basale glomérulaire (MBG). Une lésion des glomérules a pour conséquence une augmentation de la perméabilité des capillaires pour les protéines (protéinurie). Les principales manifestations du syndrome néphrotique incluent protéinurie (> $3 \mathrm{~g} / 24 \mathrm{~h}$ ), hypoalbuminémie, œdèmes, hyperlipidémie (hypercholestérolémie et hypertriglycéridémie), et lipidurie. La néphropathie diabétique (ND), la néphropathie à lésions glomérulaires minimes (LGM), la glomérulosclérose segmentaire et focale (FSGS), et la glomérulopathie extra-membraneuse (GEM) [11], maladies classées parmi les néphropathies glomérulaires [1], sont les causes majeures du syndrome néphrotique. Malgré l'identification de protéines structurelles clés pouvant contribuer à des défauts de la filtration rénale, la plupart des mécanismes à l'origine du syndrome néphrotique ne sont toujours pas élucidés. Nos travaux ont récemment montré que la protéine Angiopoietinlike-4 (Angptl4) pourrait jouer un rôle important dans certains mécanismes responsables du syndrome néphrotique.

\section{Présentation de l'Angiopoietin-like-4 (Angpt14)}

Angptl4 est une glycoprotéine sécrétée appartenant à la famille des protéines angiopoietin-like. Elle est exprimée par le tissu adipeux, le foie, le muscle squelettique, le cœur, et le placenta. C'est un inhibiteur connu de la lipoprotéine lipase (LPL), et donc un facteur d'hypertriglycéridémie [2]. Elle joue également un rôle dans la formation des métastases [3], et est un facteur anti-apoptotique pour les cellules endothéliales vasculaires $[4,12](\rightarrow)$. ANGPTL4 est un gène cible des peroxisome proliferator-activa-

$(\rightarrow)$ Voir la Nouvelle de C. Bouleti et al., page 608 de ce numéro ted receptors (PPAR) $\alpha$ [5] et $\gamma[6]$, et la majorité de l'Angptl4 circulante chez les rongeurs est sécrétée par le foie sous une forme clivée, liée aux lipoprotéines de haute densité [7]. Nos travaux ont montré une augmentation de l'expression d'Angpt|4, au niveau des podocytes et dans le sérum, dans différents modèles animaux et chez des patients atteints de syndrome néphrotique.

Angptl4 glomérulaire : implication dans le développement de la protéinurie Angptl4 est très fortement surexprimée au niveau glomérulaire dans la néphropathie à lésions glomérulaires minimes, et cela avant même l'apparition de la protéinurie. Nous avons montré, dans des modèles animaux mais également chez des patients atteints de néphropathie à lésions glomérulaires minimes, qu'Angptl4 est exprimée dans les podocytes, puis est sécrétée et traverse la membrane basale glomérulaire. Nous avons alors créé des rats transgéniques surexprimant Angptl4 spécifiquement au niveau des podocytes pour étudier le rôle de cette molécule dans le syndrome néphrotique [8]. Ces rats présentent plusieurs caractéristiques de néphropathie à lésions glomérulaires minimes: protéinurie sélective et de niveau néphrotique (albuminurie augmentée de plus de 500 fois), perte de charges de la membrane basale glomérulaire et effacement des pédicelles des podocytes. Nous avons montré dans le modèle animal PAN (puromycin aminonucleoside) [9], un modèle expérimental de néphropathie à lésions glomérulaires minimes humaine, que cette augmentation de l'expression d'Angptl4 correspondait en fait à une augmentation de deux formes d'Angpt/4 : une forme sialylée (normosialylée) et une forme présentant un déficit de sialylation (hyposialylée). Nous avons cherché à déterminer l'implication possible de ce défaut de sialylation d'Angptl4 dans le phénomène de protéinurie. Pour répondre à cette question, nous avons supplémenté des rats transgéniques en $N$-acetyl-D-mannosamine (ManNAc), un précurseur de l'acide sialique. Chez les rats supplémentés en ManNAc, la sialylation de la protéine augmente et la protéinurie est réduite de plus de $40 \%$. Nous avons ensuite voulu savoir si l'absence d'Angptl4 pouvait avoir un effet bénéfique sur la protéinurie. Nous avons donc induit un modèle expérimental de syndrome néphrotique chez des souris knock-out Angptl4 (Angptl4 ${ }^{-/-}$), et montré que chez ces souris, la protéinurie était significativement réduite comparée à celle de souris contrôles. Ces résultats nous ont donc permis de 


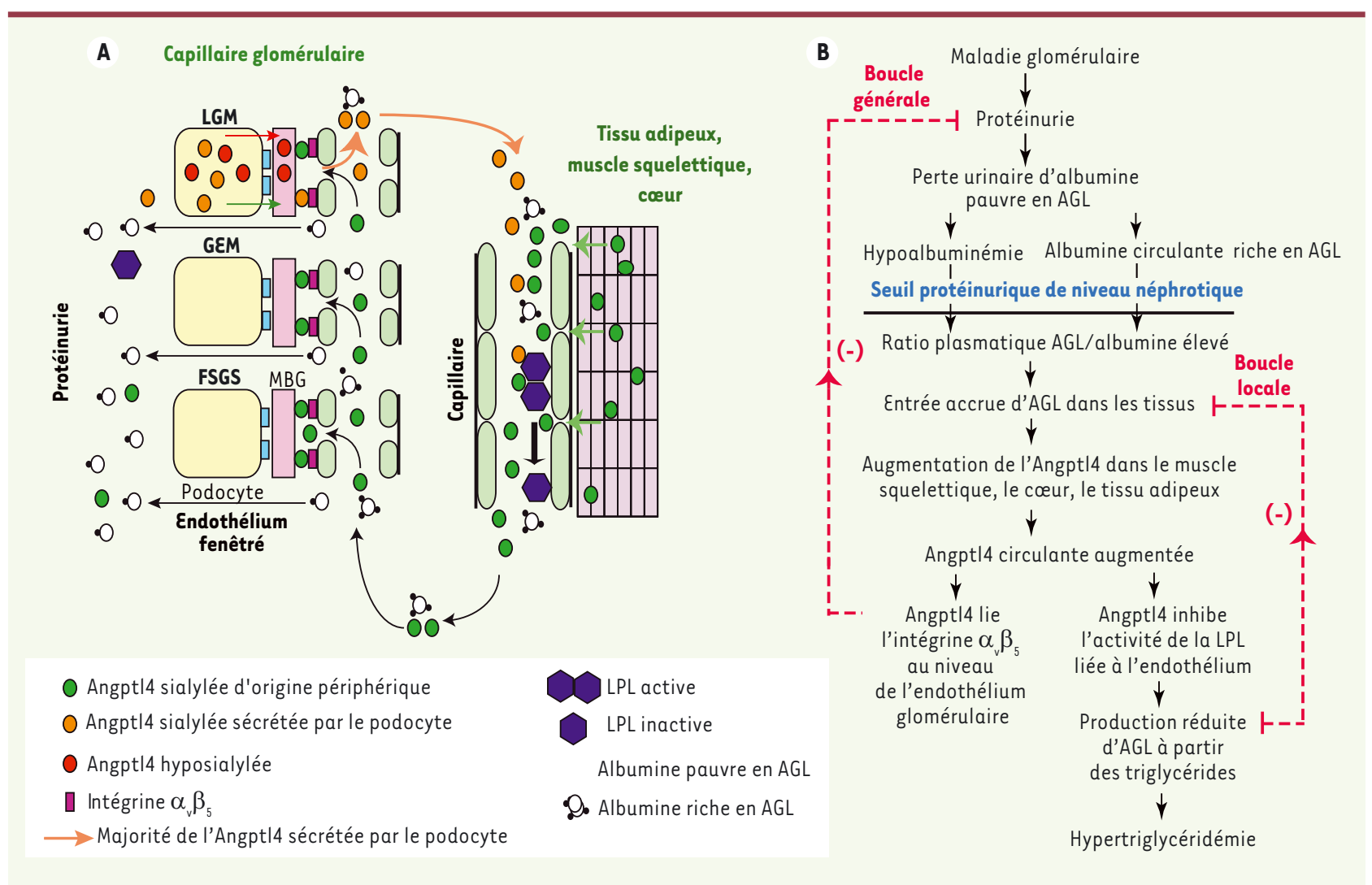

Figure 1. Rôles d'Angptl4 dans le syndrome néphrotique. A. Schéma représentant la production de la protéine Angpt/4 circulante et ses effets biologiques. Cette forme circulante d’Angpt|4, normosialylée, est sécrétée par des tissus périphériques (principalement muscle squelettique, cœur et tissu adipeux) dans les néphropathies à lésions glomérulaires minimes (LGM), glomérulosclérose segmentaire et focale (FSGS), et glomérulopathie extra-membraneuse (GEM). De plus, dans la néphropathie à lésions glomérulaires minimes, les podocytes sécrètent deux formes d’Angpt|4: une forme hyposialylée qui reste confinée dans le rein et induit une protéinurie, et la forme normosialylée qui entre dans la circulation. L’Angpt/4 circulante se lie à l'intégrine $\alpha_{v} \beta_{5}$ au niveau de l'endothélium glomérulaire pour réduire la protéinurie, ou inactive la LPL au niveau du muscle squelettique, du cœur et du tissu adipeux pour réduire l'hydrolyse des triglycérides plasmatiques en AGL, induisant une hypertriglycéridémie. Une partie d’Angpt|4 et de la LPL (lipoprotéine lipase) est perdue dans les urines. B. Schéma illustrant les boucles de régulation liant la protéinurie, I'hypoalbuminémie et l'hypertriglycéridémie médiées par Angpt|4 et les AGL (acides gras libres). Les AGL plasmatiques sont liés à l'albumine par des liaisons non covalentes, et à cause d'une perte privilégiée d'albumine pauvre en AGL lors de la protéinurie, l'albumine riche en AGL est retenue dans la circulation. Lors de la progression de la maladie glomérulaire et de l'augmentation de la protéinurie, une hypoalbuminémie se développe. Cette hypoalbuminémie, associée à la rétention de l'albumine riche en AGL, entraîne une augmentation du rapport plasmatique AGL sur albumine. Ces AGL disponibles étant augmentés, ils pénètrent dans le muscle squelettique, le cœur et le tissu adipeux pour induire une augmentation de l'expression d’Angpt|4, médiée au moins en partie par les PPAR. L’Angpt|4 sécrétée par ces tissus participe à deux boucles de régulation. Dans la boucle générale, elle se lie à l'intégrine $\alpha_{v} \beta_{5}$ au niveau de l'endothélium glomérulaire et réduit la protéinurie. Dans la boucle locale, elle inhibe l'activité de la LPL dans ces mêmes tissus périphériques pour réduire le captage des acides gras libres, court-circuitant ainsi le stimulus menant à l'augmentation de son expression (adapté de [10]).

conclure qu'Angptl4 joue un rôle clé dans le syndrome néphrotique, et que la sialylation de la protéine est un facteur important. En effet, en présence d'une augmentation importante de l'expression d'Angptl4 au niveau du podocyte, la cellule serait dans l'incapacité de sialyler la totalité de l'Angptl4 sécrétée. Cette forme hyposialylée d'Angptl4 serait responsable de l'implication de la protéine dans la protéinurie.

\section{Angptl4 circulante : diminution}

de la protéinurie mais développement d'une hypertriglycéridémie

Chez les patients atteints de certaines maladies rénales (néphropathie à lésions glomérulaires minimes, glomérulosclérose segmentaire et focale, et glomérulopathie extra-membraneuse), la concentration plasmatique d'Angptl4 est plus importante que chez les sujets sains. Nous avons montré que c'était également le cas dans différents modèles animaux. Nous avons donc cherché à élucider le rôle de l'Angptl4 circulante dans le syndrome néphrotique en utilisant des rats 
transgéniques surexprimant Angptl4 spécifiquement au niveau du tissu adipeux et sécrétant la protéine dans la circulation générale [10]. Ces rats n'ont pas de protéinurie, mais ils développent une hypertriglycéridémie. Celle-ci est absente lorsque le syndrome néphrotique est induit chez des souris Angpt/4 $4^{-/-}$, soulignant le rôle important de la protéine dans le développement de cette hypertriglycéridémie. Nous avons montré que des organes périphériques (tissu adipeux, muscle squelettique, cœur, foie) répondent à une augmentation de la protéinurie et du rapport acides gras libres ( $A G L$ )/albumine dans le sang par une surexpression d'Angptl4 circulante. Cette protéine circulante va tout d'abord se lier à l'intégrine $\alpha_{v} \beta_{5}$ au niveau de l'endothélium glomérulaire pour induire une boucle de régulation générale dont le but est de contrer la protéinurie. En effet, le blocage de l'interaction Angptl4-intégrine $\alpha_{v} \beta_{5}$, ou bien l'absence d'Angptl4 ou de l'intégrine $\alpha_{v} \beta_{5}$ (modèles animaux knock-out), ralentissent la diminution de la protéinurie. L'Angptl4 circulante participe également à une boucle de régulation locale répondant à sa propre surexpression. Elle inhibe l'activité de la lipoprotéine lipase, ce qui réduit l'hydrolyse des triglycérides en AGL qui, moins disponibles, ne seront alors plus à même d'induire l'expression d'Angptl4 par ces tissus périphériques. Dans le but de réduire la protéinurie tout en évitant le développement d'une hypertriglycéridémie, nous avons construit des formes mutantes d'Angptl4. Leur efficacité est remarquable puisqu'une seule injection permet de réduire la protéinurie de $65 \%$ pendant une durée de deux semaines chez les rats néphrotiques, des modèles de glomérulosclérose segmentaire et focale et de néphropathie diabétique, sans développement d'hypertriglycéridémie.

\section{Conclusion et perspectives}

Nous avons donc montré qu'Angptl4 contribue à la protéinurie lorsqu'elle est hyposialylée et sécrétée par les podocytes, qu'elle s'y oppose lorsqu'elle est sialylée et circulante, et qu'elle fait le lien, à travers deux boucles de régulation, entre protéinurie et hypertriglycéridémie dans le syndrome néphrotique (Figure 1). Les thérapies actuellement utilisées pour traiter les désordres liés à la protéinurie ont été empruntées à d'autres domaines de la médecine, et elles ne sont que partiellement efficaces. La découverte d'un rôle mécanistique central joué par deux formes différentes d'Angptl4 dans les maladies glomérulaires humaines et expérimentales ouvre de nouvelles stratégies de traitement. Les interventions sur ces deux formes d'Angptl4 sont prometteuses et représentent une nouvelle génération d'agents thérapeutiques pour les maladies rénales liées à des désordres glomérulaires. $\diamond$

\section{Role of Angptl4 in nephrotic syndrome:} a two-faced protein
LIENS D'INTÉRÊT

Les auteurs déclarent n'avoir aucun lien d'intérêt concernant les données publiées dans cet article.

\section{RÉFÉRENCES}

1. Habib R. Classification anatomique des néphropathies glomérulaires. Päd Fortbildk Praxis 1970 ; 28 : 3-47.

2. Yoshida K, Shimizugawa T, Ono M, Furukawa H. Angiopoietin-like 4 is a potent hyperlipidemiainducing factor in mice and inhibitor of lipoprotein lipase. J Lipid Res 2002; 43 : 1770-2.

3. Padua D, Zhang XH, Wang $Q$, et al. TGF $\beta$ primes breast tumor for lung metastasis seeding through angiopoietin-like 4. Cell 2008 ; 133 : 66-77.

4. Kim I, Kim HG, Kim H, et al. Hepatic expression, synthesis and secretion of a novel fibrinogen/angiopoietinrelated protein that prevents endothelial-cell apoptosis. Biochem J 2000 ; 346: 603-10.

5. Kersten S, Mandard S, Tan NS, et al. Characterization of the fasting-induced adipose factor FIAF, a novel peroxisome proliferator-activated receptor target gene. J Biol Chem 2000 ; 275 : 28488-93.

6. Yoon JC, Chickering TW, Rosen ED, et al. Peroxisome proliferator-activated receptor $\gamma$ target gene encoding a novel angiopoietin-related protein associated with adipose differentiation. Mol Cell Biol $2000 ; 20: 5343-9$.

7. Mandard S, Zandbergen F, van Straten $\varepsilon$, et al. The fasting-induced adipose factor/angiopoietin-like protein 4 is physically associated with lipoproteins and governs plasma lipid levels and adiposity. J Biol Chem 2006 ; 281 : 934-44.

8. Clement LC, Avila-Casado C, Macé C, et al. Podocytesecreted angiopoietin-like-4 mediates proteinuria in glucocorticoid-sensitive nephrotic syndrome. Nat Med $2011 ; 17: 117-22$.

9. Chugh SS, Clement LC, Macé C. New insights into human minimal change disease : lessons from animal models. Am J Kidney Dis 2012 ; 59 : 284-92.

10. Clement LC, Macé C, Avila-Casado C, et al. Circulating angiopoietin-like 4 links proteinuria with hypertriglyceridemia in nephrotic syndrome. Nat Med $2014 ; 20: 37-46$.

11. Girard C, Seitz-Polski B, Dolla G, et al. Nouveaux rôles physiopathologiques pour le réceptuer PLA2RI dans le cancer et la glomérulonéphrote extramembraneuse. Med Sci (Paris) 2014 ; $30: 519-25$.

12. Bouleti C, Mathivet T, Lapergue B, et al. Accident vasculaire cérébral : protection de l'intégrité vasculaire lors de la reperfusion. Med Sci (Paris) $2014 ; 30: 608-10$.

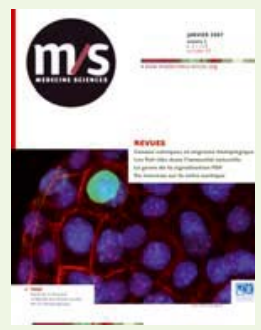

